\title{
Mental health, intimate partner violence and HIV
}

\author{
N Woollett, ${ }^{1}$ MA (Psychology, Art Therapy); A M Hatcher, ${ }^{1,2}$ MPhil (Sociology) \\ ${ }^{1}$ Wits Reproductive Health and HIV Institute, Faculty of Health Sciences, University of the Witwatersrand, Johannesburg, South Africa \\ ${ }^{2}$ School of Public Health, Faculty of Health Sciences, University of the Witwatersrand, Johannesburg, South Africa
}

Corresponding author: N Woollett (woollettn@gmail.com)

Intimate partner violence (IPV) and HIV are intersecting epidemics in South Africa (SA). Despite recognition that IPV and HIV are bidirec tionally linked, less attention has been given to mental health - a key health condition that is at the nexus of both violence and HIV/AIDS. While SA healthcare professionals have made great strides in treating HIV, the corresponding conditions of IPV and mental health receive far less clinical care. Treating mental health has the potential to positively effect HIV care and treatment, but is also a powerful gateway to enhanced comprehensive health in patients. Improving skills in managing the mental health of patients will lead to better health for them and quality of life for affected families. It can also assist health systems to deal more effectively with complex cases that so rarely achieve positive health outcomes.

S Afr Med J 2016;106(10):969-972. DOI:10.7196/SAMJ.2016.v106i10.11410

\section{Intersecting epidemics}

One in three women worldwide reports physical and/or sexual violence from an intimate partner. ${ }^{[1]}$ Intimate partner violence (IPV) poses a significant public health problem in South Africa (SA). Associated with health risk behaviours and increased use of medical services, ${ }^{[2]}$ it is linked to high rates of HIV infection among young girls and women. ${ }^{[3]}$

Mental health is a major underlying condition that exacerbates the comorbidities of IPV and HIV, and may be an important mediator between IPV and HIV outcomes. In the general population, the 12-month prevalence of any Diagnostic and Statistical Manual of Mental Disorders, 4th edition (DSM-IV)/Composite International Diagnostic Interview (CIDI) mental disorder in a national epidemiological study was $16.5 \%$, with $26.2 \%$ of respondents classified as severe cases and an additional $31.1 \%$ as moderately severe cases, indicating that mental health problems affect a large portion of the population. ${ }^{[4]}$ The most pervasive mental health consequences of IPV and HIV in the SA context tend to be depression, post-traumatic stress disorder (PTSD) and alcohol/substance use disorders. ${ }^{[4-6]}$ It is also likely that all three disorders are comorbid in this population, requiring a nuanced treatment plan that integrates management of all through one feasible intervention.

\section{The golden thread:}

A focus on mental health Mental health is the common element linking HIV and IPV (Fig. 1). Poor mental health is a symptom of both violence and HIV, but it may also serve as a mediator between the two conditions.

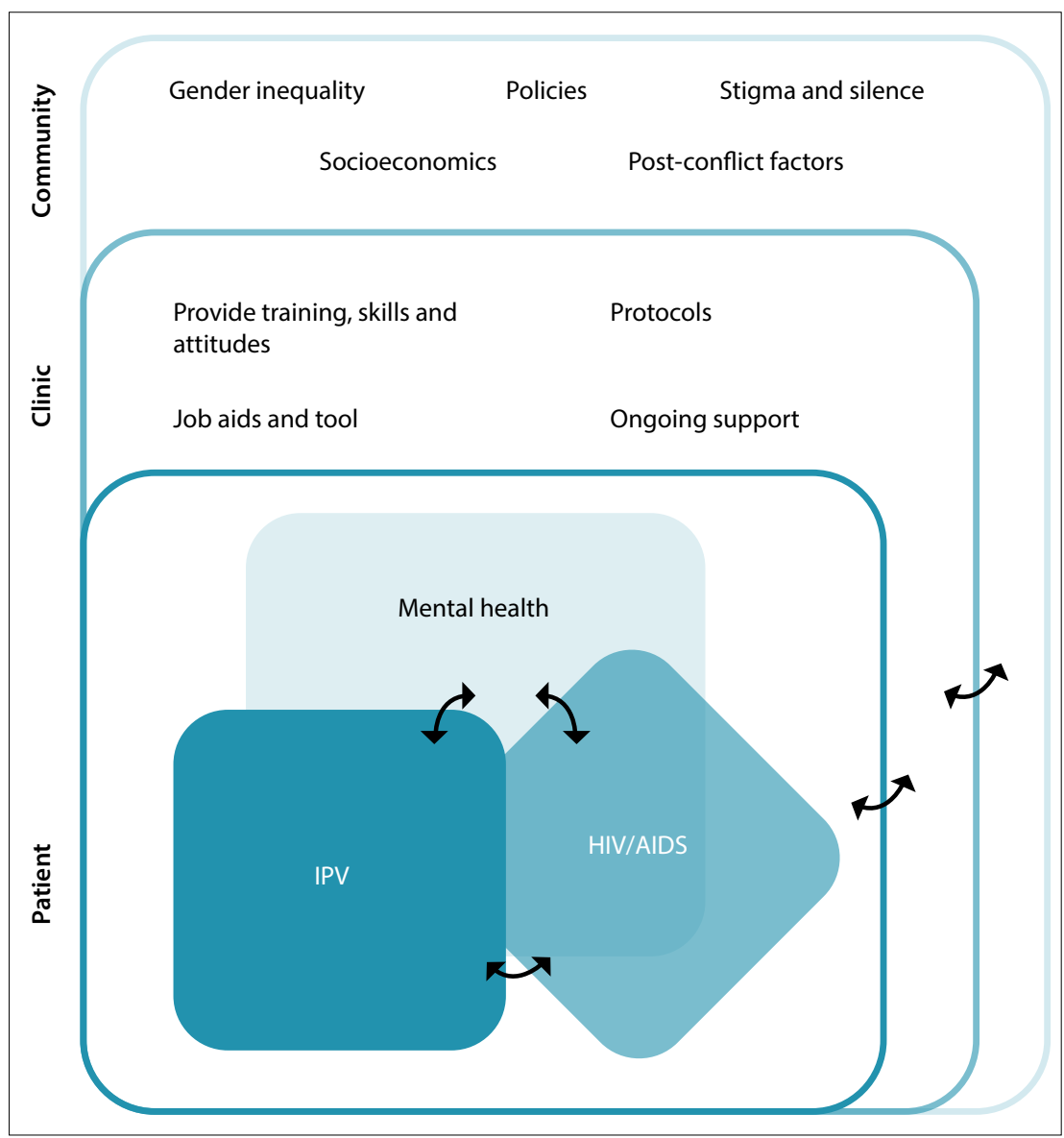

Fig. 1. Conceptual framework linking mental health to HIV and IPV.

There is a bidirectional relationship between HIV and mental health. In SA studies, high rates of depression and PTSD have been reported among individuals with HIV. ${ }^{[7]}$ Complex reasons for this include premorbid mental conditions, effects of the virus on the central nervous system, psychologi- cal impact of living with HIV/AIDS and disclosure, side-effects of medication, and consequent social stigma and discrimination. ${ }^{[8]}$ Conversely, research indicates that women with mental health problems, particularly comorbid drinking and substance use problems, are at heightened risk for HIV, 
primarily as a result of high sexual risk-taking behaviour. ${ }^{[9]}$ Common mental disorders compromise quality of life and functional outcomes in HIV-positive individuals. ${ }^{[5]}$ Furthermore, traumatic stress, or exposure to traumatic events/situations, whereby individuals are overwhelmed by an inability to cope with the experience, accelerates disease progression in HIV-infected individuals by suppressing immune functioning. ${ }^{[5]}$ Addressing traumatic stress and its mental health burden through effective and timeous interventions offers an opportunity to make a transformational impact on the HIV epidemic and patients' lives.

Similarly, there is a bidirectional relationship between IPV and mental health. Traumatic stress is the primary mechanism by which IPV might cause subsequent depression, suicide attempts and PTSD. ${ }^{[5,6]}$ These common mental health problems tend to be most significantly related to IPV. Equally, research suggests that women with mental health difficulties are more likely to experience violence victimisation, particularly those with depression and PTSD. Both conditions are highly prevalent common mental health problems in SA. ${ }^{[4,6]}$

Lastly, there is a bidirectional relationship between HIV and IPV. The latter leads to an increased risk of incident HIV infection among women, ${ }^{[10]}$ and male perpetrators of violence are more likely to be HIV positive, thereby further increasing the possibility of transmission. ${ }^{[11]}$ Conversely, HIV leads to violence because it causes relationship conflict - disclosing one's HIV status to a partner leads to arguments and blaming one person for 'bringing' the disease into the relationship. ${ }^{[12]}$ Among women already living with HIV, experiencing IPV halves their odds of treatment adherence. ${ }^{[13]}$

An important evolution of the thinking around trauma has come with the notion of continuous trauma, which attempts to define traumatic stress when there is a psychological impact that is real and ongoing, with current threat and danger. ${ }^{[14]}$ This is a deviation from the more common understanding of PTSD that implicitly manages the effects of trauma in the past, with an acceptance that the threat is no longer current and real. Although some people who have experienced traumatic events in the past may currently reside in an objectively safe environment, this is not the case in contexts of protracted civil conflict, mass displacement, enduring IPV or high levels of community violence. In these circumstances, ongoing threat and danger is an inescapable part of daily life. Such conditions affect millions of people worldwide, particularly in post-conflict settings such as SA. Continuous traumatic stress therefore offers a particularly valuable local clinical dimension, with concomitant treatment recognising the ongoing nature of the traumatic experience. ${ }^{[14]}$

It is important to note that IPV has mental health consequences for both victims and their communities. A recent nationally representative study in SA found that increased depressive symptoms among women are associated with an increase in the perceived frequency of neighbourhood domestic violence, highlighting the communitylevel mental health effects of such violence. ${ }^{[15]}$ In addition, mental health treatment alleviates the suffering of victims, and recent studies suggest that the treatment itself may reduce IPV, mental problem symptoms, and risk of future violent victimisation, even if women do not leave their violent partners. ${ }^{[16]}$

\section{Recommendations with regard to clinical skills}

Both IPV and mental disorders are common, but unfortunately remain largely unrecognised and untreated within the health system. ${ }^{[1]}$ Considering that patients are more likely to seek healthcare for HIV than for mental health or IPV, providers of HIV care are an optimal entry point for identifying these patients. Interventions to manage both mental health and IPV need to be simple enough to be incorporated into existing standard packages of care in HIV treatment settings. Interventions should be brief, culturally adaptable, and scalable to settings where task-shifting models of care can be employed.

\section{Clinical concepts}

Several underlying ideas inform how mental health and IPV care are delivered.

Non-judgemental, caring support is essential for stigmatised health challenges such as IPV and mental health. Women who experience violence and mental health problems need health professionals to provide front-line support: kindness, attentive listening, sensitive non-judgemental enquiry about their needs, validation of women's disclosure without pressure, and help with access to referrals - all in a safe and containing encounter. ${ }^{[1,17]}$ This approach is consistent with 'psychological first aid', a first response to individuals undergoing traumatic events. ${ }^{[1]}$ Arguably, this is not a difficult undertaking and falls in line with ethical codes of conduct for health professionals. Unfortunately, however, such skills are scarce owing to provider attitudes, lack of values clarification, gender inequitable norms, personal experience of violence, mental health difficulties, and HIV. ${ }^{[18]}$

Trauma-informed care is central to safe, effective delivery of mental health and IPV treatment. Typically, victims are in a state of shock and sometimes experiencing traumatic stress when health professionals encounter them. The definition of trauma is underscored by the event having caused intense fear, helplessness or horror. Patients have a strong need to feel safe. Clinicians must increase feelings of safety by asking what women need to help them feel safe.

Privacy and confidentiality are paramount. Often these comprise a kind demeanour, and especially for children/adolescents it may be having a known 'safe person' near them. Simply ask to find out.

Patient-centred care is optimal in treating mental problems, IPV and HIV. With this approach the client's priorities and preferences lead the clinical treatment plan. In cases of IPV, for example, it is not helpful to inform victims that they should leave the perpetrator or escape dangerous situations. IPV victims are aware of this and are the experts of their own relationships. Clinicians should provide information about the client's options and offer services, not 'fix the problem.

\section{Clinical interventions for mental health}

A number of interventions can be provided at the clinic for mental health treatment:

- In recent years, effective models for feasible and acceptable mental health treatment in low-resource settings have been developed using paraprofessionals and trained community counsellors to deliver established psychotherapies adapted for local settings. Most of these models are group based to improve effect, and tend to be aligned with evidence-based, trauma-focused cognitive behavioural therapy interventions. There is also consistent mentorship and supervision for those providing care. ${ }^{[15]}$

- Meditation and mindfulness-based practices can be used as a means of managing what is often experienced as uncontrollable anxiety responses.

- Psycho-education crucially involves the validation of responses and experiences as context related and, to a large degree, context appropriate.

- Anxiety management and some cognitive restructuring elements are recommended for the treatment of continuous traumatic stress. ${ }^{[14]}$ The objective of cognitive restructuring is to assist patients to endure 


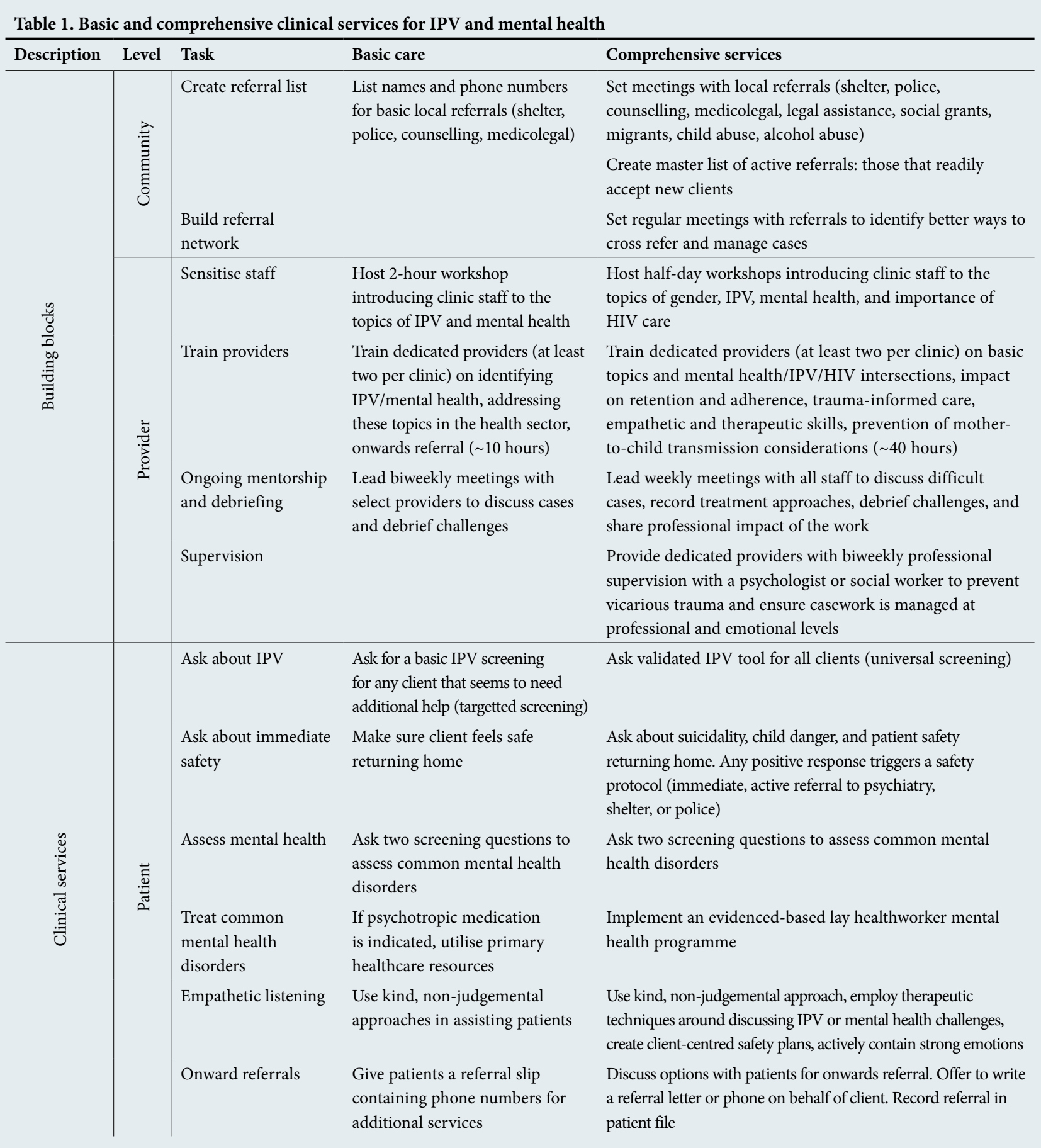

living in such contexts without becoming totally hopeless, apathetic and disengaged or antisocial and highly aggressive.

- Social support, in the form of carefully facilitated support groups or therapeutic sessions, is a critical factor in ameliorating the effect of trauma. Health professionals are well positioned to offer support to victims who come into their care.

\section{Clinical interventions for IPV}

While IPV interventions are in their infancy, the following promising practices are being tested worldwide:

- Post-exposure prophylaxis (PEP) for HIV after rape or sexual assault must be provided by healthcare professionals as part of post-rape care for victims who are HIV negative (see PEP Guidelines ${ }^{[19]}$ ).
- IPV screening at the clinic coupled with empowerment counselling and referrals may reduce further experience of violence. ${ }^{[20,21]}$ Our team is currently testing an intervention, i.e. Safe \& Sound in antenatal care (trial registered at ISRCTN: 35969343). Based on lessons from Safe \& Sound, Table 1 provides a summary of the clinical services required for basic and comprehensive care for IPV and mental health. It simultaneously illustrates how to create a basic package of IPV services within a clinic setting or how to offer comprehensive IPV care.

- Health worker home visits around the time of pregnancy can reduce the experience of violence for up to 2 years post partum. ${ }^{[22]}$ The Domestic Violence Enhanced (DOVE) home visitation programme consists of six brochure-driven sessions, each lasting 15 - 25 minutes. ${ }^{[2]}$ Home visits are led by a combination of lay and professional healthcare workers. 
Male perpetrators of IPV have a high prevalence of HIV and mental disorders, particularly alcohol use disorders, depression and PTSD. ${ }^{[23]}$ While the search for effective mental healthcare strategies to reduce recidivism among perpetrators is ongoing, treatment of alcohol use disorders is emerging as a method of reducing male perpetration. ${ }^{[15]}$

\section{Non-clinical interventions for IPV}

At a population level, interventions that address the underpinning societal gender norms that perpetuate IPV against women and girls are essential.

\section{Supporting staff to tackle mental health and IPV}

Often, clinic staff are limited in their abilities to help patients effectively if they are working in chaotic, stressful and unsupportive environments. The mental health of staff is hugely important, as they often confront sensitive and difficult issues that may precipitate emotional distress, defensiveness or self-disclosure. Management of these factors can take a heavy toll on their own wellbeing.

Curbing potential vicarious trauma in health professionals through regular debriefing, targeted training in mental healthcare, diversity in case loads and recognition of the difficulty of the work by management is recommended. Also facilitating a supportive, efficient and utilised employee assistance programme for healthcare providers through the National Department of Health is recommended.

\section{Conclusion}

Healthcare professionals, especially those engaged with HIV-positive patients, are uniquely placed to identify mental health problems and IPV and to promote early intervention and appropriate intersectoral referral. This will possibly also improve retention in care and adherence to treatment - all fundamental health system goals. Implementing this approach is a powerful opportunity to improve job satisfaction among health professionals.

Addressing mental health problems in patients will have positive effects on other areas of health, such as HIV and IPV, leading to relief of suffering in patients and engaging the powerful positive potential of the healthcare system. Interventions to reduce IPV at the neighbourhood level could positively have an impact on other dimensions of population and economic health, including healthcare costs, economic productivity, and broader gender equity.
1. Garcia-Moreno C, Hegarty K, Lucas d'Oliveira AF, et al. The health-systems response to violence against women. Lancet 2014:385(9977):1567-1579. DOI:10.1016/s0140-6736(14)61837-7

2. Gass JD, Stein DI, Williams DR, et al. Intimate partner violence, health behaviours, and chronic physical illness among South African women. S Afr Med J 2010;100(9):582-585.

3. Jewkes RK, Dunkle K, Nduna M, Shai N. Intimate partner violence, relationship power inequity, and
. . Jewkes RK, Dunkle K, Nduna M, Shai N. Intimate partner violence, relationship power inequity, and
incidence of HIV infection in young women in South Africa: A cohort study. Lancet 2010;376(9734):41-48. incidence of HIV infection in young wo

4. Williams DR, Herman A, Stein DJ, et al. Twelve-month mental disorders in South Africa: Prevalence, service use and demographic correlates in the population-based South African Stress and Health Study. Psychol Med 2008;38(2):211-220. DOI:10.1017/s0033291707001420

5. Seedat S. Interventions to improve psychological functioning and health outcomes of HIV-infected individuals with a history of trauma or PTSD. Curr HIV/AIDS Rep 2012;9(4):344-350. DOI:10.1007/s1 1904-012-0139-3

6. Devries KM, Mak JY, Bacchus LJ, et al. Intimate partner violence and incident depressive symptoms and suicide attempts: A systematic review of longitudinal studies. PLoS Med 2013;10(5):1-11. DOI:10.1371/journal.pmed.1001439

7. Myer L, Smit J, Roux LL, et al. Common mental disorders among HIV-infected individuals in South Africa: Prevalence, predictors and validation of brief psychiatric rating scales. AIDS Patient Care STDs 2008;22(2):147-158. DOI:10.1089/apc.2007.0102

8. Freeman M, Nkomo N, Kafaar Z, et al. Factors associated with prevalence of mental disorders in people living with HIV/AIDS in South Africa. AIDS Care 2007;19(10):1201-1209. DOI:10.1080/09540120701426482

9. Pitpitan EV, Kalichman SC, Eaton LA, et al. Gender-based violence and HIV sexual risk behavior:

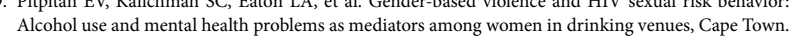
Alcohol use and mental health problems as mediators among women in drin
Soc Sci Med 2012;75(8):1417-1425. DOI:10.1016/j.socscimed.2012.06.020

10. Li Y, Marshall CM, Rees HC, et al. Intimate partner violence and HIV infection among women: A systematic review and meta-analysis. J Int AIDS Soc 2015;17(1):1-12. DOI:10.7448/ias.17.1.1884

11. Dunkle KL, Decker MR. Gender-based violence and HIV: Reviewing the evidence for links and causal pathways in the general population and high-risk groups. Am J Repro Immunol 2012;69:1-7. DOI:10.1111/aji.12039

12. Colombini M, James $\mathrm{C}, \mathrm{Ndwiga} \mathrm{C}$, et al.The risks of partner violence following HIV status disclosure, and health service responses: Narratives of women attending reproductive health services in Kenya. J Int AIDS Soc 2016;19(1):1-7. DOI:10.7448/ias.19.1.20766

13. Hatcher AM, Smout EM, Turan JM, Christofides N, Stoeckl H. Intimate partner violence and engagement in HIV care and treatment among women: A systematic review and meta-analysis. AIDS 2015;29(16):2183-2194. DOI:10.1097/qad.0000000000000842

14. Kaminer D, Eagle G, Crawford-Browne S. Continuous traumatic stress as a mental and physical health challenge: Case studies from South Africa. J Health Psych 2016;21(1):1-12. OI: $10.1177 / 1359105316642831$

15. Meffert SM, McCulloch CE, Neylan TC, et al. Increase of perceived frequency of neighborhood domestic violence is associated with increase of women's depression symptoms in a nationally representative longitudinal study in South Africa. Soc Sci Med 2015;131:89-97. DOI:10.1016/.jsocscimed.2015.03.008

16. Iverson KM, Gradus JL, Resick PA, et al. Cognitive-behavioral therapy for PTSD and depression symptoms reduces risk for future intimate partner violence among interpersonal trauma survivors. J Consult Clin Psychol 2011;79(2):193-202. DOI:10.1037/a0022512

17. Joyner K, Theunissen L, de Villiers L, et al. Emergency care provision for, and psychological distress in, survivors of domestic violence. S Afr Fam Pract 2007;49(3):15-15d. DOI:10.1080/20786204.2007.10873523

18. Jina R, Jewkes R, Christofides N, et al. Knowledge and confidence of South African health care providers regarding post-rape care: A cross-sectional study. BMC Health Ser Res 2013:13(257):1-12. DOI:10.1186/1472-6963-13-257

19. Moorhouse M, Bekker LG, Black V, et al. Guideline on the management of occupational and nonoccupational exposure to the human immunodeficiency virus and recommendations for post-exposure prophylaxis: 2015 Update. S Afr J HIV Med 2015;16(1):1-14. DOI:10.4102/saihivmed.v16i1.399

20. Kiely M, El-Mohandes AA, El-Khorazaty MN, Blake SM, Gantz MG. An integrated intervention Kiely M, El-Mohandes AA, El-Khorazaty MN, Blake SM, Gantz MG. An integrated intervention
to reduce intimate partner violence in pregnancy: A randomized controlled trial. Obstet Gynecol to reduce intimate partner violence in pregnancy: A random

21. Tiwari A, Leung WC, Leung TW, Humphreys J, Parker B, Ho PC. A randomised controlled trial of empowerment training for Chinese abused pregnant women in Hong Kong. Br J Obstet Gynaecol 2005;112(9):1249-1256. DOI:10.1111/j.1471-0528.2005.00709.x

22. Sharps PW, Bullock LF, Campbell JC, et al. Domestic violence enhanced perinatal home visits: The DOVE randomized clinical trial. J Women's Health (Larchmt) 2016, May 20. [Epub ahead of print] DOI:10.1089/jwh.2015.5547

23. Machisa MT, Christofides N, Jewkes R. Structural pathways between child abuse, poor mental health outcomes and male-perpetrated intimate partner violence (IPV). PLoS ONE 2016;11(3):e0150986. DOI:0.1371/journal.pone.0150986 